\section{OPEN ACCESS}

Edited by:

Marie-Louise Van Der Hoorn, Leiden University Medical

Center, Netherlands

Reviewed by:

Johannes Duvekot,

Erasmus MC, Netherlands

Huri Güvey,

Private PARKHAYAT Hospital, Turkey

*Correspondence:

Chloe A. Brady

chloe.brady@manchester.ac.uk

Specialty section:

This article was submitted to

Obstetrics and Gynecology,

a section of the journal

Frontiers in Medicine

Received: 04 August 2021 Accepted: 23 September 2021

Published: 18 October 2021

Citation:

Brady CA, Williams C, Batra G, Church E, Tower CL, Crocker IP and Heazell AEP (2021) Immunomodulatory Therapy Reduces the Severity of Placental Lesions in Chronic Histiocytic Intervillositis. Front. Med. 8:753220

doi: 10.3389/fmed.2021.753220

\title{
Immunomodulatory Therapy Reduces the Severity of Placental Lesions in Chronic Histiocytic Intervillositis
}

\author{
Chloe A. Brady ${ }^{1 *}$, Charlotte Williams ${ }^{1,2}$, Gauri Batra ${ }^{3}$, Elaine Church ${ }^{4}$, Clare L. Tower ${ }^{4}$, \\ Ian P. Crocker ${ }^{1}$ and Alexander E. P. Heazell ${ }^{1,4}$ \\ 'Tommy's Maternal and Fetal Health Research Centre, St Mary's Hospital, The University of Manchester, Manchester, \\ United Kingdom, ${ }^{2}$ Exeter Medical School, University of Exeter, Exeter, United Kingdom, ${ }^{3}$ Pediatric Histopathology, Central \\ Manchester University Hospitals National Health Service Foundation Trust, Manchester, United Kingdom, ${ }^{4}$ Saint Mary's \\ Hospital Managed Clinical Maternity Service, Manchester Academic Health Science Centre, Manchester, United Kingdom
}

Chronic histiocytic intervillositis $(\mathrm{CHI})$ is a rare, but highly recurrent inflammatory placental lesion wherein maternal macrophages infiltrate the intervillous space. Pregnancies with $\mathrm{CHI}$ are at high risk of fetal growth restriction, miscarriage or stillbirth. Presently, the diagnosis can only be made after histopathological examination of the placenta. Given its proposed immunological etiology, current treatments include aspirin, heparin, and immunomodulatory agents. However, the rationale for these medications is largely based upon small case series and reports as there is a lack of larger studies investigating treatment efficacy. Therefore, this study sought to determine whether inclusion of immunomodulatory medications was effective at reducing the severity of lesions and improving pregnancy outcomes in subsequent pregnancies. Thirty-three women with a history of $\mathrm{CHI}$ in at least one pregnancy (index case) were identified retrospectively through medical records. Twenty-eight participants presented with a first subsequent pregnancy and a further 11 with a second subsequent pregnancy at a specialist clinic for pregnancy after loss. Data on maternal demographics, medical history, medication, pregnancy outcome, and placental pathology was collected and compared between pregnancies. Twenty-seven (69\%) subsequent pregnancies were treated with at least one or both of prednisolone and hydroxychloroquine. Inclusion of at least one immunomodulatory agent in treatment regimen resulted in an almost $25 \%$ increase in overall livebirth rate (61.5 vs. $86.2 \%)$. In women treated with immunomodulatory medication a greater proportion of placentas had reduced severity of lesions compared to those treated without (86.7 vs. 33.3\%, respectively). A reduction in $\mathrm{CH}$ severity was associated with a $62.3 \%$ improvement in livebirth rate compared to those where severity remained unchanged in relation to the index case. These data provide preliminary evidence that the use of immunomodulatory medication in the management of $\mathrm{CHI}$ improves histopathological lesions and the chance of livebirth in subsequent pregnancies. Due to $\mathrm{CHI}$ 's rarity and ethical and feasibility issues, 
randomized controlled trials in affected women are challenging to conduct. As a result, collaboration between centers is required in future to increase study sample sizes and elucidate the mechanisms of hydroxychloroquine and prednisolone in reducing pathology.

Keywords: placental histopathology, prednisolone, hydroxychloroquine, stillbirth, treatment, miscarriage, outcomes

\section{INTRODUCTION}

Chronic histiocytic intervillositis (CHI), also known as chronic intervillositis or chronic intervillositis of unknown etiology (1), is a pregnancy disorder strongly associated with fetal growth restriction, miscarriage, stillbirth and neonatal death (2-5). Estimated to affect 6 in every 10,000 pregnancies over 12 weeks' gestation, $\mathrm{CHI}$ is characterized by maternal macrophage infiltration into the intervillous space of the placenta and has a $25-100 \%$ risk of recurrence in subsequent pregnancies $(1,3,6,7)$. Many cases also exhibit marked perivillous and/or intervillous fibrin deposition and trophoblast necrosis (8). Due to its asymptomatic nature and a lack of reliable associated biomarkers, currently a diagnosis of CHI can only be made following delivery by histopathological examination of the placenta. Management of CHI is further complicated by a lack of standardized treatment options proven to prevent recurrence.

Owing to the presence of maternal macrophages and the reported increased incidence in women with autoimmune disease, $\mathrm{CHI}$ has been hypothesized to be a disorder of failed maternal-fetal tolerance and excessive inflammation (9). On this basis and due to the presence of intervillous and perivillous fibrin, current treatments include thromboprophylactic agents such as aspirin and low-molecular-weight heparin (LMWH) as well as those aimed at suppressing inflammation e.g., corticosteroids and hydroxychloroquine (9). A systematic review of six observational studies conducted in 2010 found no significant improvement in pregnancy outcome with aspirin and LMWH alone. Though a growing number of case reports detail use of immunomodulatory agents such as prednisolone and hydroxychloroquine $(9,10)$, there remains a striking lack of larger studies supporting the efficacy of any treatment regime in reducing severity of $\mathrm{CHI}$ and improving pregnancy outcomes. Notably, a prior case series has highlighted that women with worse obstetric histories tend to be prescribed more therapeutic agents, despite their unproven efficacy (9). Due to the high rate of recurrence of $\mathrm{CHI}$ and severe consequences of the disorder, studies are urgently needed to determine effective therapies.

By retrospectively identifying women with a previous diagnosis of $\mathrm{CHI}$, we aimed to investigate pregnancy outcomes and placental pathology in subsequent pregnancies referred to a specialist service following poor perinatal outcome, stillbirth or neonatal death. We hypothesized, due to the immunological nature of $\mathrm{CHI}$, that treatment regimens where immunomodulatory agents were included would decrease the severity of the condition and consequently improve the chance of livebirth.
MATERIALS AND METHODS

\section{Participant Recruitment and Data Collection}

Women with a previous histopathological diagnosis of $\mathrm{CHI}$ between 2009 and 2021 were identified retrospectively from medical records at Manchester University NHS Foundation Trust, UK. The majority of these cases were identified after the death of a baby or late miscarriage when histopathological evaluation of the placenta is recommended practice. In other cases (following the birth of a live infant) the placenta is sent away for examination for a variety of clinical indications (e.g., FGR, fetal compromise at birth, placental abruption, and previous late pregnancy loss). CHI was diagnosed by a specialist perinatal pathologist in accordance with its initial description by Labarrere and Mullen as a placental lesion consisting of histiocytic (macrophage) infiltration into the intervillous space (8). Data on maternal demographics, medical history including results of tests for autoantibodies (lupus anticoagulant, antiphospholipid, antinuclear, and anticardiolipin antibodies) and obstetric history were collected from the woman's case record. The first pregnancy diagnosed with CHI was classified as the "index" case, with data on any subsequent pregnancies recorded where applicable from retrospective medical records. Pregnancy outcomes consisted of liveborn and still living, liveborn at term and still living (>37 weeks' gestation), miscarriage (fetal death $<24$ weeks gestation, including spontaneous abortion), stillbirth (fetal death $>24$ weeks gestation), and termination of pregnancy for fetal anomaly (TOPFA). Cases of neonatal death (death of an infant within 28 days after birth) were also recorded. Fetal growth restriction (FGR) was defined as growth below the $3 \mathrm{rd}$ percentile, and small for gestational age (SGA) as between 3rd and 10th centile (11). Centiles were calculated for pregnancies $>20$ weeks' gestation using the GROW centile calculator, for cases where maternal demographics, pregnancy outcome and fetal sex were known (12).

The therapeutic agents used in our service evolved over time following publications from other researchers $(3,9,10,13)$ and following discussion with colleagues with expertise in lupus in pregnancy. Initially, pregnancies with $\mathrm{CHI}$ were managed using Aspirin and LMWH, but this evolved to a combination of Aspirin was given at a dose of $75-150 \mathrm{mg}$ once a day, a prophylactic dose of LMWH (e.g., Tinzaparin 4,500iu) once a day, Hydroxychloroquine $200 \mathrm{mg}$ twice a day and Prednisolone $20 \mathrm{mg}$ once a day in the morning. Drug therapy was started from a viability scan at 6-7 weeks' gestation. Women underwent ultrasound assessment of uterine artery Doppler at 17 weeks' gestation, if this showed no abnormality then Prednisolone was 
reduced by $5 \mathrm{mg}$ per week. If there was evidence of uterine artery notching or raised pulsatility index Prednisolone was continued at $20 \mathrm{mg}$ and then uterine artery Doppler's were reassessed after 2 weeks. If there was no improvement by 21 weeks' gestation the Prednisolone was reduced at this stage. Women underwent regular ultrasound assessment of fetal growth, amniotic fluid volume and umbilical artery Doppler after 23 week's gestation at a minimum frequency of 3-weekly intervals.

Where available, placental histopathology reports were analyzed for detail on histopathological features including the presence of villitis, increased fibrin deposition and recurrence and severity of $\mathrm{CHI}$. Change in severity of $\mathrm{CHI}$ in subsequent pregnancies was in comparison to the severity of the index case of CHI. Focal CHI without accompanying fibrin deposition was classified as "mild," those with accompanying fibrin as "moderate," and diffuse, high-grade lesions classified as "severe."

Informed and written consent was obtained from all study participants and ethical approval granted by the NRES Committee North West-Greater Manchester West and NRES Committee London-City \& East.

\section{Statistical Analysis}

Statistical analysis was conducted using GraphPad Prism Version 9.1.0. Chi-square and Kruskal-Wallis tests were performed for categorical and continuous variables, respectively. For statistical analysis, first and second subsequent pregnancies were combined, and pregnancy outcomes divided into liveborn and still living or liveborn at term and still living vs. adverse outcome (TOPFA, miscarriage, stillbirth and neonatal death). Where sample number precluded analysis by Chi-square, Fisher's Exact test was performed instead. Statistical analysis of treatment regimen was performed by dividing participants into those which received immunomodulatory therapy (one or both of prednisolone and hydroxychloroquine) and those without (one or both of aspirin and heparin, or untreated).

\section{RESULTS}

\section{Participant Demographics}

Thirty-three women with a history of at least one pregnancy affected by CHI between 2009 and 2021 were identified retrospectively from medical records. Twenty-eight women presented with a subsequent pregnancy and 11 of these with a further second subsequent pregnancy. Participant demographics, medical and obstetric history are shown in Table 1. Four Dichorionic-Diamniotic (DCDA) twin pregnancies were included in the study. Twenty-six women were White British (78.8\%), with a smaller proportion of Asian $(n=6)$ and Black African women $(n=1)$. Four index CHI pregnancies (12.1\%) were conceived using ART, compared to only one first subsequent pregnancy (3.6\%) and no second subsequent pregnancies. Underlying autoimmune disease was present in four cases (12.1\%), consisting of coeliac disease, hypothyroidism, autoimmune thrombocytopenia, and hypermobility syndrome, respectively. Seventeen women had been tested for antinuclear antibodies, three of which were positive (17.7\%) and 25 and 22 women had testing for anti-cardiolipin antibodies and
TABLE 1 | Demographics and obstetric and medical history of women with a diagnosis of chronic histiocytic intervillositis $(\mathrm{CHI})$.

\begin{tabular}{|c|c|c|c|}
\hline & Index CHI & $\begin{array}{c}\text { 1st subsequent } \\
\text { pregnancy }\end{array}$ & $\begin{array}{c}\text { 2nd } \\
\text { subsequent } \\
\text { pregnancy }\end{array}$ \\
\hline$N$ participants & 33 & 28 & 11 \\
\hline$N$ fetuses & 34 & 30 & 12 \\
\hline $\begin{array}{l}\text { Maternal age } \\
\text { (years) }\end{array}$ & 32 (19-38) & $34(22-41)$ & $37(26-40)$ \\
\hline BMl & $26(20-47)$ & $27(20-47)$ & $27(20-40)$ \\
\hline \multicolumn{4}{|l|}{ Ethnicity } \\
\hline White British & 26 (78.8\%) & & \\
\hline Asian & $6(18.2 \%)$ & & \\
\hline Black African & $1(3.0 \%)$ & & \\
\hline \multicolumn{4}{|l|}{ Lifestyle } \\
\hline Smoker & $3(9.4 \%)$ & 1 (3.6\%) & 0 \\
\hline Unknown & 1 & & \\
\hline $\begin{array}{l}\text { Alcohol } \\
\text { consumption }\end{array}$ & 0 & & \\
\hline Unknown & 1 & & \\
\hline \multicolumn{4}{|l|}{ Obstetric history } \\
\hline Previous livebirths & $0(0-5)$ & & \\
\hline Previous losses & $0(0-4)$ & & \\
\hline Primigravida & $11(33.3 \%)$ & & \\
\hline ART pregnancy & $4(12.1 \%)$ & $1(3.6 \%)$ & 0 \\
\hline Twin pregnancy & $1(3.0 \%)$ & $2(7.1 \%)$ & 1 (9.1\%) \\
\hline \multicolumn{4}{|l|}{ Medical history } \\
\hline $\begin{array}{l}\text { Autoimmune } \\
\text { disease }\end{array}$ & $4(12.1 \%)$ & & \\
\hline $\begin{array}{l}\text { Pre-existing } \\
\text { hypertension }\end{array}$ & $2(6.1 \%)$ & & \\
\hline $\begin{array}{l}\text { Anti-nuclear } \\
\text { antibodies }\end{array}$ & $3(17.7 \%)$ & & \\
\hline Untested & 16 & & \\
\hline $\begin{array}{l}\text { Lupus } \\
\text { anticoagulant }\end{array}$ & $1(3.9 \%)$ & & \\
\hline Untested & 7 & & \\
\hline
\end{tabular}

Index pregnancy was defined as a participant's first pregnancy diagnosed with $\mathrm{CHI}$ by placental histopathological examination following poor outcome. Subsequent pregnancies refer to those following diagnosis. Continuous variables are presented as median (range) and categorical variables $N$ (percentage). ART, assisted reproductive technology; BMI, body mass index.

anti-phospholipid antibodies, respectively, none of which were positive. Of 26 women tested for lupus anticoagulant, one was positive (3.9\%). Pre-existing hypertension was present in two women $(6.1 \%)$.

\section{Pregnancy Outcomes}

Outcomes of index pregnancies diagnosed with $\mathrm{CHI}$ and subsequent pregnancies are listed in Table 2. The proportion of infants born by Cesarean section increased from $27.3 \%(6 / 33)$ in index pregnancies to $59.1 \%(13 / 28)$ and $45.5 \%(5 / 11)$ in first and second subsequent pregnancies, respectively, though this was not statistically significant. Fetal sex did not differ significantly across pregnancies, and in two subsequent pregnancies fetal sex had not been determined due to loss early in gestation. 
TABLE 2 | Pregnancy outcomes of index and subsequent pregnancies in women with a diagnosis of chronic histiocytic intervillositis $(\mathrm{CHI})$.

\begin{tabular}{|c|c|c|c|}
\hline & Index CHI & $\begin{array}{l}\text { 1st subsequent } \\
\text { pregnancy }\end{array}$ & $\begin{array}{c}\text { 2nd } \\
\text { subsequent } \\
\text { pregnancy }\end{array}$ \\
\hline$N$ participants & 33 & 28 & 11 \\
\hline$N$ fetuses & 34 & 30 & 12 \\
\hline $\begin{array}{l}\text { Cesarean section } \\
\text { (>24 weeks) }\end{array}$ & $6(27.3 \%)$ & $13(59.1 \%)$ & $5(45.5 \%)$ \\
\hline Male fetus & 13 (38.2\%) & $5(53.6 \%)$ & $7(58.3 \%)$ \\
\hline Unknown fetal sex & 0 & 2 & 0 \\
\hline \multicolumn{4}{|c|}{ Pregnancy outcome } \\
\hline $\begin{array}{l}\text { Liveborn and still } \\
\text { living }\end{array}$ & $4(11.8 \%)$ & $21(70.0 \%)$ & $12(100 \%)$ \\
\hline Stillbirth & $16(47.1 \%)$ & 1 (3.3\%) & 0 \\
\hline Miscarriage & $5(14.7 \%)$ & $6(20.0 \%)$ & 0 \\
\hline TOPFA & $5(14.7 \%)$ & $1(3.3 \%)$ & 0 \\
\hline Neonatal death & $4(11.8 \%)$ & $1(3.3 \%)$ & 0 \\
\hline $\begin{array}{l}\text { Gestation at } \\
\text { delivery (weeks) }\end{array}$ & $26(17-41)$ & $37(12-39)$ & 38 (35-39) \\
\hline Birthweight centile & $2.8(0-68.4)$ & $22.8(0.4-99.9)$ & $\begin{array}{c}23.2 \\
(3.8-89.6)\end{array}$ \\
\hline \multicolumn{4}{|l|}{ Complications } \\
\hline FGR <3rd centile & 13 (52.0\%) & $3(13.6 \%)$ & 0 \\
\hline $\begin{array}{l}\text { SGA 3rd-10th } \\
\text { centile }\end{array}$ & $5(20.0 \%)$ & $2(9.1 \%)$ & $1(9.1 \%)$ \\
\hline $\begin{array}{l}\text { Preterm <37 } \\
\text { weeks ( } \% \text { of } \\
\text { livebirths) }\end{array}$ & $6(75.0 \%)$ & $5(22.7 \%)$ & $3(25.0 \%)$ \\
\hline \multicolumn{4}{|c|}{ Maternal comorbidities } \\
\hline $\begin{array}{l}\text { Gestational } \\
\text { diabetes }\end{array}$ & $1(3.0 \%)$ & 0 & 0 \\
\hline
\end{tabular}

Index pregnancy was defined as a participant's first pregnancy diagnosed with $\mathrm{CHI}$ by placental histopathological examination following poor outcome. Subsequent pregnancies refer to those following diagnosis. Miscarriage was defined as fetal death $<24$ weeks gestation, and stillibirth as fetal death $>24$ weeks gestation. Neonatal death refers to the death of an infant within 28 days after birth. Continuous variables are presented as median (range) and categorical variables $N$ (percentage). FGR, fetal growth restriction; SGA, small for gestational age; TOPFA, termination of pregnancy for fetal anomaly.

The outcome of subsequent pregnancy/ies improved significantly, with $70 \%$ of first and $100 \%$ of second subsequent pregnancies resulting in a liveborn and still living infant, compared to $11.8 \%$ of index pregnancies $(p<0.0001)$. Gestation at delivery also increased significantly across pregnancies $(p=0.002)$, from a median of 26 weeks' gestation in index cases to 37 weeks' gestation in first subsequent $(p=0.04)$ and 38 weeks in second subsequent pregnancies $(p=0.002)$. Of infants who were liveborn, rates of neonatal death were significantly reduced from $50 \%(4 / 8)$ in index cases to $3.0 \%$ $(1 / 33)$ of subsequent pregnancies $(p=0.003)$. TOPFA occurred in five index pregnancies (14.7\%) and one first subsequent pregnancy (3.3\%), after diagnosis of skeletal dysplasia $(n=1)$, severe FGR $(n=3)$, triploidy $(n=1)$ and neurological disorder $(n=1)$. In one case of stillbirth occurring in an index pregnancy, Trisomy 18 was diagnosed at post-mortem. For infants whose birthweight centiles could be calculated, rates of FGR $<3 \mathrm{rd}$
TABLE 3 | Histopathology of placentas from index and subsequent pregnancies in women with chronic histiocytic intervillositis $(\mathrm{CHI})$.

\begin{tabular}{lccc}
\hline Index CHI & $\begin{array}{c}\text { 1st subsequent } \\
\text { pregnancy }\end{array}$ & $\begin{array}{c}\text { 2nd } \\
\text { subsequent } \\
\text { pregnancy }\end{array}$ \\
\hline $\boldsymbol{N}$ placentas & 34 & 30 & 12 \\
$\boldsymbol{N}$ placentas with & $34(100 \%)$ & $23(76.7 \%)$ & $8(66.7 \%)$ \\
pathology reports & $34(100 \%)$ & $11(47.8 \%)$ & $2(25.0 \%)$ \\
CHI & $5(14.7 \%)$ & $6(26.1 \%)$ & $2(25.0 \%)$ \\
$\begin{array}{l}\text { Chronic villitis } \\
\text { Increased fibrin }\end{array}$ & $18(52.9 \%)$ & $7(30.4 \%)$ & $2(25.0 \%)$ \\
deposition & & & $1(12.5 \%)$ \\
$\begin{array}{l}\text { Small for } \\
\text { gestational age }\end{array}$ & $7(20.6 \%)$ & $4(17.4 \%)$ &
\end{tabular}

Index pregnancy was defined as a participant's first pregnancy diagnosed with $\mathrm{CHI}$ by placental histopathological examination following poor outcome. Subsequent pregnancies refer to those following diagnosis. Variables are expressed as $N$ (percentage).

centile decreased significantly between index and subsequent pregnancies from $52.0 \%(13 / 25)$ to $9.1 \%(3 / 33)(p=0.0007)$. There were no significant differences in the incidence of SGA across pregnancies. Overall birthweight centiles increased significantly across pregnancies from a median of 2.8 in index cases to 22.8 and 23.2 in first and second subsequent pregnancies, respectively ( $p<0.0001$ ). In subsequent pregnancies, $23.5 \%$ of livebirths were preterm (8/34), significantly reduced from $75.0 \%$ $(6 / 8)$ of index cases $(p=0.01)$. One participant was diagnosed with gestational diabetes in their index pregnancy (3.0\%), and there were no cases of preeclampsia. Chorioamnionitis occurred in one index case (3.0\%) alongside $\mathrm{CHI}$, however since $\mathrm{CHI}$ recurred without infection in the participant's subsequent pregnancy this case was included in the study.

\section{Placental Pathology}

Findings of placental histopathology are shown in Table 3. By design, all index pregnancies had an accompanying placental pathology report. The number of cases with histopathological examination of the placenta decreased to $76.7 \%(23 / 30)$ and $66.7 \%(8 / 12)$ in first and second subsequent pregnancies as a result of placentas not having been sent for histopathological examination. The incidence of CHI significantly decreased from index pregnancies, with an overall recurrence rate of $41.9 \%$ $(13 / 31)$ in subsequent pregnancies $(p<0.0001)$. There were no significant differences in the incidence of villitis, increased fibrin deposition and placentas classified as small for gestational age. Pregnancies with concurrent $\mathrm{CHI}$ and villitis in the placenta exhibited no differences in outcome compared to those with CHI alone.

The severity of CHI lesions in placentas from index and subsequent pregnancies as determined by histopathological examination is shown in Figure 1. Detail of CHI lesion severity was available for $28 / 34(82.4 \%)$ and $30 / 31$ (96.8\%) placentas from index and subsequent pregnancies, respectively. Placentas from index cases exhibited mainly mild or moderate $\mathrm{CHI}[39.3 \%$ $(11 / 28)$ and $39.3 \%(11 / 28)]$, followed by a smaller proportion 


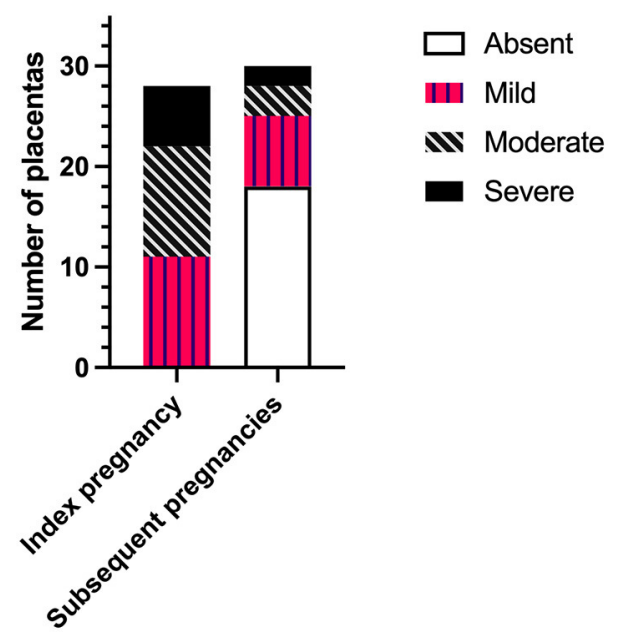

FIGURE 1 | Severity of chronic histiocytic intervillositis (CHI) lesions in placentas from index and subsequent pregnancies according to pathologist's report. Index $\mathrm{CH}$ refers to a participant's first pregnancy diagnosed with $\mathrm{CHI}$ by placental histopathological examination following poor outcome. Subsequent pregnancies refer to those following diagnosis. of severe cases $[21.4 \%(6 / 28)$. In comparison, CHI was absent in the majority of placentas from subsequent pregnancies $(60 \%$ (18/30)], whilst $23.3 \%$ displayed mild lesions (7/30), $10.0 \%$ moderate (3/30), and $6.7 \%$ severe (2/30). Overall, severity of CHI was significantly reduced in subsequent pregnancies compared to index $(p<0.0001)$.

\section{Treatment Effect}

Treatment regimen across pregnancies is shown in Figure 2. The majority of index pregnancies had no medication $(24 / 33,72.7 \%)$, compared to $10.7 \%(3 / 28)$ of first subsequent pregnancies. No second subsequent pregnancies were untreated. In index pregnancies, the nine treated participants were on medication for pre-existing medical conditions or risk factors and took aspirin alone $(n=4)$, in combination with LMWH $(n=4)$, or hydroxychloroquine $(n=1)$. There was no significant difference in the livebirth rate of index pregnancies between women on medication and those without. In subsequent pregnancies, $69.2 \%$ (27/39) of all participants received immunomodulatory therapy with at least one or both of hydroxychloroquine or prednisolone, with the majority of participants 53.9\% (21/39) receiving all four medications. All four women with a positive autoantibody screen were treated in their subsequent pregnancies $(n=5)$ with aspirin and LMWH $(n=1)$; aspirin, low-molecular-weight heparin and hydroxychloroquine $(n=1)$; aspirin, prednisolone, and hydroxychloroquine $(n=1)$ or a combination of all four medications $(n=2)$. These pregnancies all resulted in the birth of a live infant surviving past 28 days of life.

The overall rate of infants liveborn and still living (including infants born $<37$ weeks' gestation) was almost $25 \%$ higher in women receiving immunomodulatory treatment with one or both of hydroxychloroquine and prednisolone compared to those without $[25 / 29(86.2 \%)$ vs. $8 / 13(61.5 \%)]$ (Figure 3A). However, this did not reach statistical significance due to the number

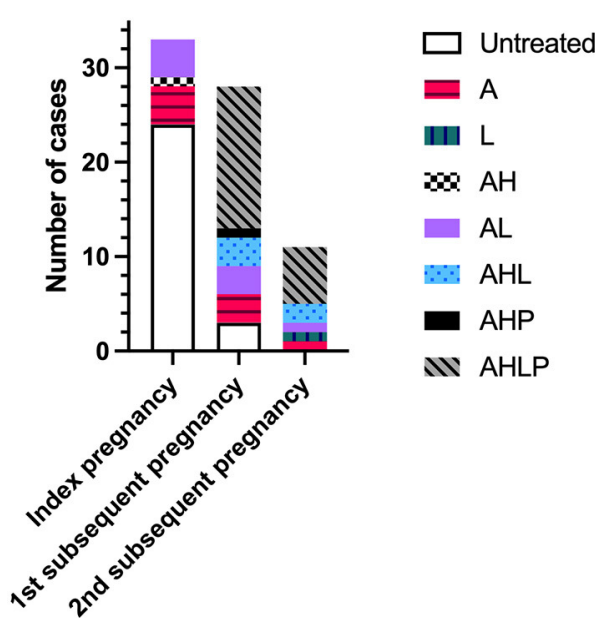

FIGURE 2 | Treatment regimen across index pregnancies with chronic histiocytic intervillositis (CHI) and first and second subsequent pregnancies. Index pregnancy was defined as a participant's first pregnancy diagnosed with $\mathrm{CHI}$ by placental histopathological examination following poor outcome. Subsequent pregnancies refer to those following diagnosis. A, aspirin; $\mathrm{H}$, hydroxychloroquine; L, low-molecular-weight heparin; P, prednisolone.

of pregnancies included in the study $(p=0.11)$. Inclusion of immunomodulatory medication in treatment regimen also resulted in a $19 \%$ increase in the proportion of infants liveborn at term (>37 weeks) (Figure 3B) [65.5\% (19/29) with immunomodulators vs. $46.2 \%(6 / 13)$ without], though again this was not statistically significant. There were no significant effects of immunomodulatory treatment on the incidence of FGR [8.33\% (2/24) with vs. $12.5 \%(1 / 8)$ without], SGA [12.5\% (3/24) with vs. $12.5 \%(1 / 8)$ without], or on overall birthweight centiles (median 20.9 with vs. 19.5 without).

Change in $\mathrm{CHI}$ severity following treatment regimen is shown in Figure 4A. 7/13 (53.8\%) placentas from women treated without immunomodulatory medication were sent for histopathological examination, compared to 23/29 of those who were treated with it $(82.8 \%)$. Change in severity of $\mathrm{CHI}$ in one subsequent pregnancy treated without immunomodulatory medication could not be determined due to a lack of detail on severity of the index case. The majority of remaining placentas from pregnancies treated without immunomodulatory medication exhibited no change in $\mathrm{CHI}$ severity compared to their index case $[66.7 \%(4 / 6)]$. In comparison, $86.7 \%(20 / 23)$ of cases treated with immunomodulators displayed a reduction in $\mathrm{CHI}$ severity or a lack of recurrence $(p=0.02)$. Decreased severity of $\mathrm{CHI}$ in subsequent pregnancies was associated with a $62.3 \%$ increase in livebirth rate compared to pregnancies where severity was unchanged $[20 / 22(90.9 \%)$ vs. $2 / 7$ (28.6\%), respectively] $(p=0.003$; Figure 4B).

\section{DISCUSSION}

These data suggest that the use of one or both of prednisolone and hydroxychloroquine in the treatment of CHI resulted in a reduction of disease severity and a trend toward an increase in livebirth rate. Overall, a decrease in CHI severity was associated 


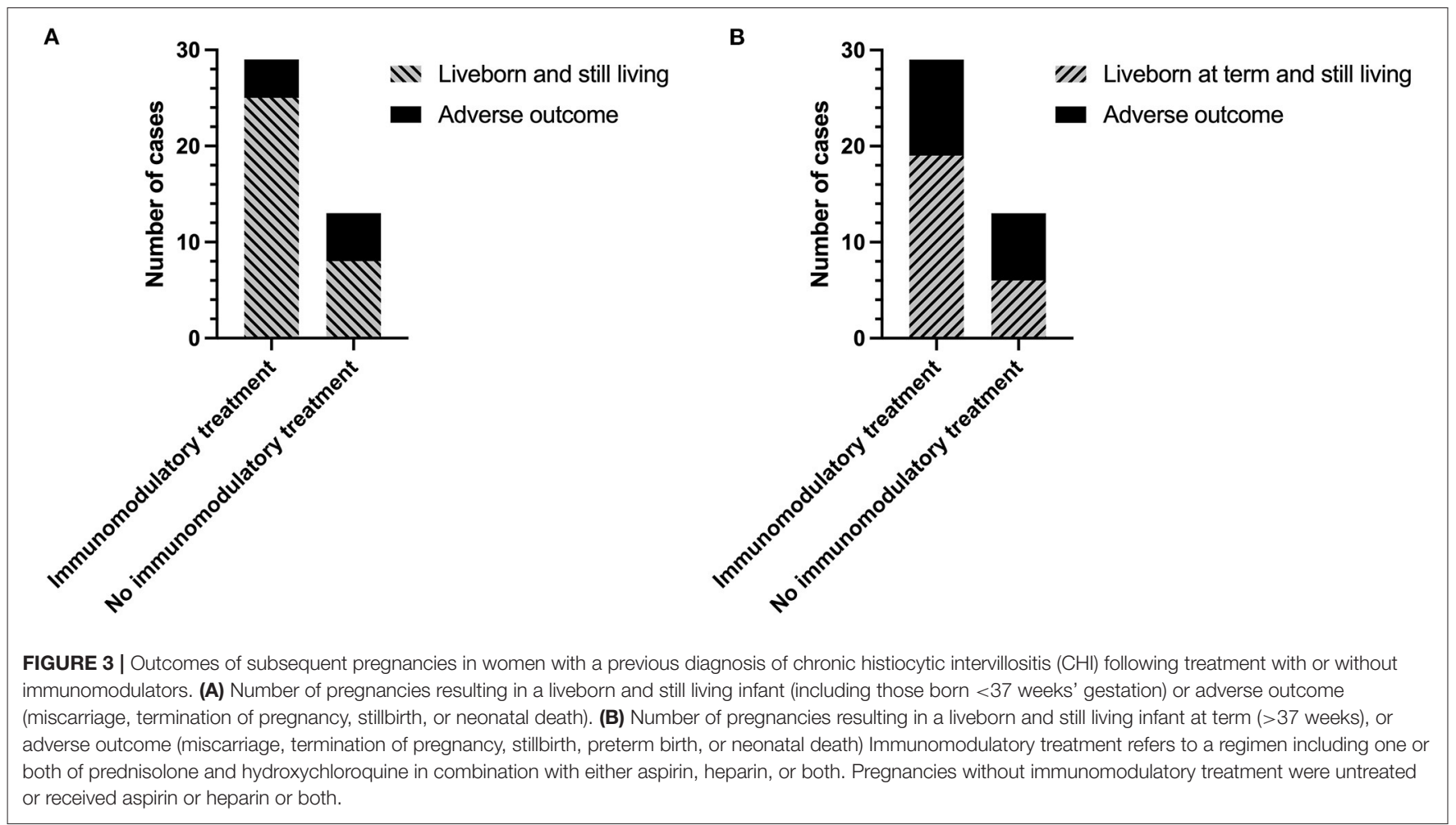

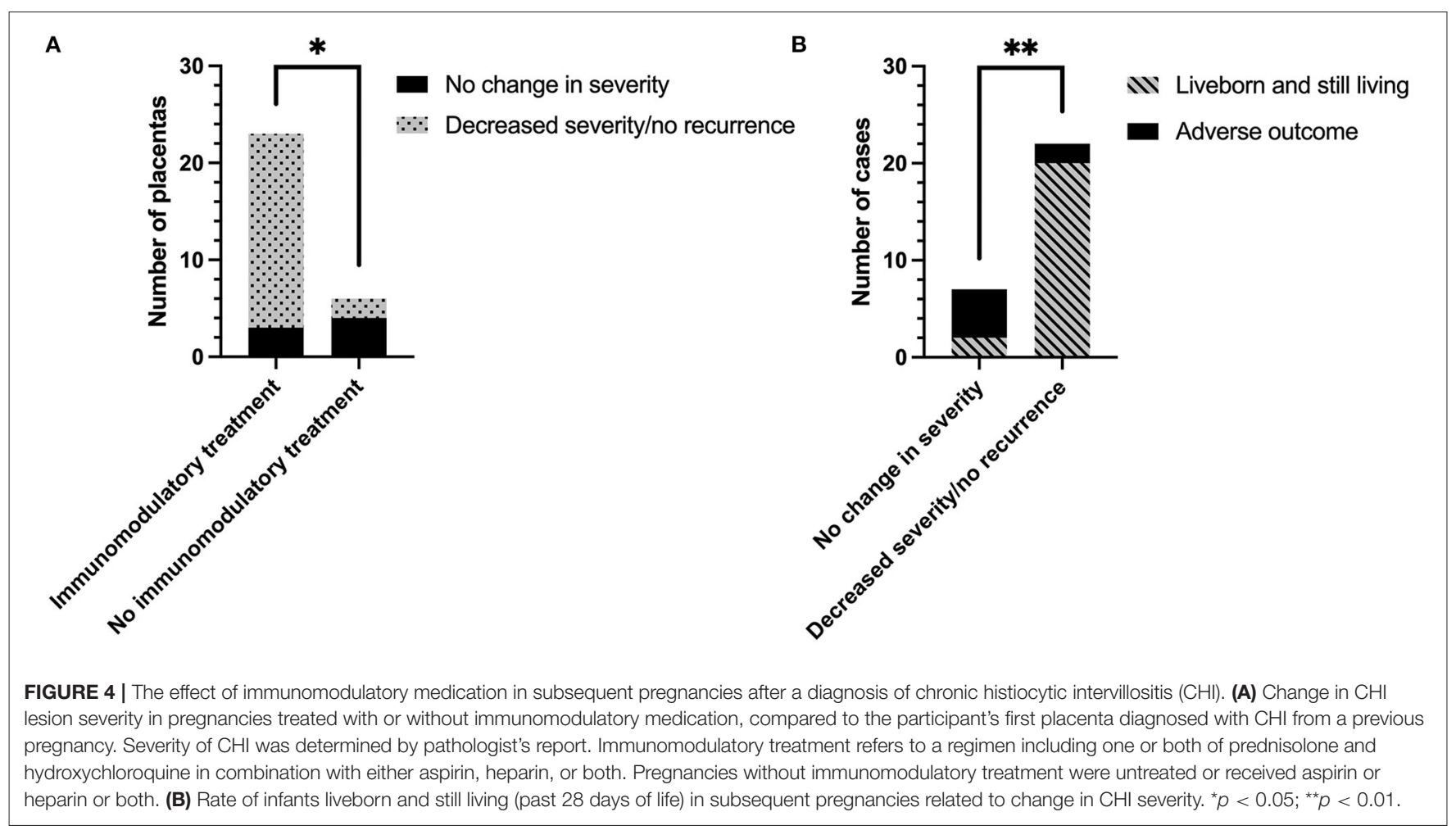


with a $62.3 \%$ reduction in pregnancy loss. These findings suggest that management of $\mathrm{CHI}$ in a specialist center for women pregnant after loss improves outcomes, with all second subsequent pregnancies resulting in the birth of a healthy infant.

\section{Strengths and Limitations}

Previously, treatment of $\mathrm{CHI}$ has been informed by case reports and small studies detailing successful pregnancy outcome following the use of prednisolone and other immunomodulatory agents (10), due to a lack of randomized controlled trials informing management. Given CHI's rarity and ethical and feasibility issues surrounding recruitment of women with such poor obstetric histories, it is unlikely that there will be a study where treatment is compared to placebo or a combination of agents known to be ineffective (e.g., aspirin and LMWH alone). As such, case series prove invaluable in guiding the care of women with a history of $\mathrm{CHI}$. To date, this series is the largest to investigate the efficacy of immunomodulatory medication in the treatment of $\mathrm{CHI}$ and proposes a standardized protocol of medication to increase the chance of positive outcome in subsequent pregnancies.

Through analysis of medical records, we have been able to follow women through subsequent pregnancies following diagnosis of $\mathrm{CHI}$ and formulate a well-characterized cohort of a rare condition. However, we recognize that women who decided to become pregnant or became pregnant could differ from all women who have $\mathrm{CHI}$. The demographic characteristics of our sample were similar to those from other case series $(5,14)$, suggesting that this potential selection bias had not significantly altered the nature of the sample.

In previous investigations, the rate of recurrence of $\mathrm{CHI}$ and accompanying outcome in treated pregnancies has been relatively well-documented, though there is a paucity of evidence regarding improvement in lesion severity. For the majority of pregnancies we included, detail of lesion severity was available in placental pathology reports, allowing any change to be related to an individual's index case. To our knowledge, this is the first time such an approach has been taken in a case series of treated pregnancies following CHI. Here, it was shown that although overall recurrence of $\mathrm{CHI}$ in participants treated with immunomodulatory treatment did not differ significantly compared to those treated without, the severity of lesions was greatly reduced. This indicates that inclusion of disease severity as an outcome in future studies on CHI may provide better insight into treatment effect rather than simple recurrence rate alone.

Due to the retrospective nature of this study, there were certain variables for which data was not available within medical records. For instance, many women whose index case of $\mathrm{CHI}$ occurred less recently had not received screening for all autoantibodies as this was a more recent practice. Additionally, the range of antibodies tested was limited, which may explain the low incidence of autoantibodies in this cohort in comparison to studies which looked more specifically at antibody status and have reported incidences between 29 and 58\% (9). Since this suggestion of an autoimmune component to $\mathrm{CHI}$, it has become standard practice within our center to offer women an antibody screen following referral. In future, this will allow better characterization of any possible role pre-existing antibodies may have within $\mathrm{CHI}$ and may inform immunosuppressive treatment if underlying conditions are present.

Several subsequent pregnancies included were without an accompanying placental histopathology report as a direct result of having not been sent for examination. In all these cases, all respective pregnancies had resulted in a livebirth. This is suggestive that there may have been a bias toward reporting pathology only in pregnancies resulting in poor outcome. In addition, pathologists were informed of patient's medical history which may have influenced diagnosis. In response to decreased rates of pathological examination in subsequent pregnancies, the importance of histopathological examination following a history of $\mathrm{CHI}$ is becoming increasingly recognized amongst midwives and clinicians within our center. Consequently, placental histopathology is now a routine requirement and is anticipated to improve sample size for prospective studies.

\section{Clinical Context}

Though the specific effects of prednisolone and hydroxychloroquine could not be individually determined here, our data is suggestive that inclusion of at least one of these medications in the treatment of $\mathrm{CHI}$ can significantly reduce the severity of macrophage infiltration into the intervillous space. Further to this, a reduction in CHI severity increased the likelihood of a live birth. This is in line with previous evidence that pregnancies where $\mathrm{CHI}$ is severe or diffuse in the placenta are more strongly associated with poor outcome (15). Pregnancy outcomes in $\mathrm{CHI}$ and severity following hydroxychloroquine use have not been well-documented previously, although a single study stated that four out of six pregnancies where it was included in treatment regimen resulted in a liveborn infant (9). Prednisolone has also in several case reports been associated with improved pregnancy outcome and reduced severity of both $\mathrm{CHI}$ and fibrin deposition compared to treatment with aspirin or heparin alone $(2,10,16)$. Within our cohort, there was no significant difference in fibrin deposition between index and subsequent pregnancies, and the majority did not have an increase in fibrin noted in their pathology report. This is perhaps unsurprising as fibrin deposition has been classified previously as an accompanying feature which may or may not occur alongside CHI (1).

Whilst the exact cause of pathology in CHI is unknown, current evidence suggests that it is driven by excessive maternal inflammation. Inflammatory features characterized so far include the presence of partner-directed T lymphocytes and antibodies (17) and deposition of complement in the placenta (18). Our observation that administration of prednisolone and hydroxychloroquine aimed at suppressing the maternal immune response reduces $\mathrm{CHI}$ severity is therefore consistent with this hypothesis. The specific mechanism of hydroxychloroquine and prednisolone's anti-inflammatory effects in CHI are unknown, though in mouse and in vitro models of antiphospholipid syndrome, it has been suggested that hydroxychloroquine is able to reduce complement activation and antibody binding to the syncytiotrophoblast (19). In addition, hydroxychloroquine does not appear to have negative effects on placental explants and 
increases release of anti-inflammatory cytokine interleukin-10 (19). As complement deposition and excessive inflammation is characteristic of $\mathrm{CHI}$, further investigation into the mechanisms of immunomodulatory therapy in the condition is warranted.

Evidence of $\mathrm{CHI}$ as an alloimmune condition has also provided rationale for the use of other immunomodulatory and immunosuppressive routes of treatment, including intravenous immunoglobulin (IVIG) therapy, tumor necrosis factor (TNF) antagonists, and tacrolimus (20). In a case report by Abdulghani et al. (13), IVIG was used following the failure of prednisolone to produce successful outcome in a previous pregnancy, and resulted in two subsequent healthy pregnancies. Histopathological examination of both placentas revealed that CHI had not recurred in either case. Similarly, use of TNF antagonist adalimumab was reported in the pregnancy of a woman with recurrent intervillositis and has been proposed as a possible agent in the prevention of recurrent miscarriage (21). Though these case reports provide anecdotal evidence hinting at the benefits of these agents, there is still a lack of larger studies to justify their use and as such much controversy remains.

Despite the lack of comparative studies where the efficacy of immunomodulatory medication in treating $\mathrm{CHI}$ has been specifically investigated, a livebirth rate of $66.7 \%$ in 21 women receiving various combinations of aspirin, heparin, prednisolone and hydroxychloroquine has previously been reported by Mekinian et al. (9). Conversely, in the only systematic review of intervention in $\mathrm{CHI}$ to date, treatment was suggested to correlate with worse outcome, with a reported livebirth rate of $30.8 \%$ (3). Here, livebirth rate with inclusion of one or both of hydroxychloroquine and prednisolone was markedly higher than both studies at $86.2 \%$. Importantly, the systematic review did not include any studies wherein hydroxychloroquine was administered, and treatment regimen was less consistent than that used within this case series, both of which may be factors in the differences observed. As in the aforementioned studies, differences in livebirth rates within this cohort were not proven to be significantly different between treatment groups, likely due to limited sample size considering that data did show a trend toward statistical significance. Given that the only systematic review is now a decade old, it is probable that an updated study of the literature is required to better characterize treatment combinations and their effect on pregnancy outcome.

In subsequent pregnancies, gestational age at delivery, fetal growth, and rates of preterm birth improved greatly, though these effects could not be significantly attributed to immunomodulatory treatment. It is possible that this is due to limited numbers, or an effect of treatment using LMWH and aspirin. Adverse outcomes may also have been reduced as a consequence of increased fetal monitoring following specialist care after previous pregnancy loss. As there were insufficient numbers of untreated women and all participants had attended the specialist clinic, it was not possible to distinguish whether these outcomes were a result of intervention or treatment. Increased fetal monitoring is unable to influence fetal growth, but delivery via Cesarean section showed a trend toward increasing across subsequent pregnancies despite lacking statistical significance. Other centers have reported low levels of spontaneous labor in cases of CHI and early delivery of a liveborn infant has been detailed in a case report following observations of fetal growth plateau $(9,10)$. Therefore, it is possible that electing for early delivery may have had a beneficial effect in select cases.

Recurrence rates of $\mathrm{CHI}$ in subsequent pregnancies vary widely between studies and have been reported between 25 and $100 \%(1,3,7)$. Within this cohort, recurrence of $\mathrm{CHI}$ was $41.9 \%$, though the majority of pregnancies were treated which in 20 placentas reduced severity, some to the point where $\mathrm{CHI}$ was absent. In agreement with observations made by other groups, recurrence of $\mathrm{CHI}$ was not always associated with adverse outcome $(9,22)$. Bos et al. in their systematic review stated that incidence and recurrence of $\mathrm{CHI}$ may vary due to differing inclusion and exclusion criteria across studies (1). A controversial criterion noted was the exclusion of cases with chronic villitis or villitis of unknown etiology alongside CHI. As concurrent $\mathrm{CHI}$ and chronic villitis lesions have been reported in $25-47 \%$ of placentas $(15,23)$, those with combined lesions were not excluded here as they represented a similar and considerable proportion of cases. Of particular interest was the observation that the incidence of chronic villitis did not differ across pregnancies and therefore seemed unaffected by intervention within the participant group studied. In addition, though chronic villitis is itself associated with FGR (24), outcomes of pregnancies with concurrent $\mathrm{CHI}$ and chronic villitis were not significantly different, suggesting that chronic villitis in the presence of $\mathrm{CHI}$ may not have been a strong factor in adverse outcome.

\section{CONCLUSION}

The data from this retrospective study suggest that including at least one of hydroxychloroquine or prednisolone in treatment regimen of pregnancies following diagnosis of $\mathrm{CHI}$ is effective at reducing placental lesion severity. In turn, decreased severity of CHI improves the likelihood of livebirth in subsequent pregnancies. From this, we propose that a standardized treatment protocol including aspirin, heparin, prednisolone and hydroxychloroquine may prove beneficial in the management of CHI. Studies into the efficacy of both hydroxychloroquine and prednisolone as well as other immunomodulatory agents used within the condition is extremely limited, and feasibility of randomized controlled trials in affected women is low given their poor obstetric history. To overcome this challenge, collaboration between centers specializing in the management of $\mathrm{CHI}$ is required to increase sample sizes to allow sufficient evaluation of treatment regimen.

\section{DATA AVAILABILITY STATEMENT}

The raw data supporting the conclusions of this article will be made available by the authors, without undue reservation.

\section{ETHICS STATEMENT}

The studies involving human participants were reviewed and approved by NRES Committee North West-Greater Manchester West and NRES Committee London-City \& 
East. The patients/participants provided their written informed consent to participate in this study.

\section{AUTHOR CONTRIBUTIONS}

$\mathrm{CB}$, IC, and $\mathrm{AH}$ contributed toward the conception and design of the study. $\mathrm{CB}$ and $\mathrm{CW}$ organized the study database. $\mathrm{CB}$, $\mathrm{AH}$, and $\mathrm{EC}$ collected the data. $\mathrm{CB}$ performed the data analysis and wrote the manuscript. $\mathrm{AH}$ and IC assisted with data analysis and data presentation. $\mathrm{GB}, \mathrm{EC}, \mathrm{CT}$, and $\mathrm{AH}$ assisted with manuscript proofreading and corrections. All authors

\section{REFERENCES}

1. Bos M, Nikkels PGJ, Cohen D, Schoones JW, Bloemenkamp KWM, Bruijn JA, et al. Towards standardized criteria for diagnosing chronic intervillositis of unknown etiology: a systematic review. Placenta. (2018) 61:80-8. doi: 10.1016/j.placenta.2017.11.012

2. Ozawa N, Yamaguchi K, Shibata M, Sugibayashi R, Yagi H, Sago H, et al. Chronic histiocytic intervillositis in three consecutive pregnancies in a single patient: differing clinical results and pathology according to treatment used. $J$ Obs Gynaecol Res. (2017) 43:1504-8. doi: 10.1111/jog.13404

3. Contro E, deSouza R, Bhide A. Chronic intervillositis of the placenta: a systematic review. Placenta. (2010) 31:1106-10. doi: $10.1016 /$ j.placenta.2010.10.005

4. Traeder J, Jonigk D, Feist H, Brocker V, Langer F, Kreipe H, et al. Pathological characteristics of a series of rare chronic histiocytic intervillositis of the placenta. Placenta. (2010) 31:1116-9. doi: 10.1016/j.placenta.2010.09.012

5. Marchaudon V, Devisme L, Petit S, Ansart-Franquet H, Vaast P, Subtil D. Chronic histiocytic intervillositis of unknown etiology: clinical features in a consecutive series of 69 cases. Placenta. (2011) 32:140-5. doi: 10.1016/j.placenta.2010.11.021

6. Boyd TK, Redline RW. Chronic histiocytic intervillositis: a placental lesion associated with recurrent reproductive loss. Hum Pathol. (2000) 31:1389-96. doi: 10.1016/S0046-8177(00)80009-X

7. Parant O, Capdet J, Kessler S, Aziza J, Berrebi A. Chronic intervillositis of unknown etiology (CIUE): relation between placental lesions and perinatal outcome. Eur J Obs Gynecol Reprod Biol. (2009) 143:9-13. doi: 10.1016/j.ejogrb.2008.06.012

8. Labarrere C, Mullen E. Fibrinoid and trophoblastic necrosis with massive chronic intervillositis: an extreme variant of villitis of unknown etiology. Am J Reprod Immunol. (1987) 15:85-91. doi: 10.1111/j.1600-0897.1987.tb00162.x

9. Revaux A, Mekinian A, Nicaise P, Bucourt M, Cornelis F, Lachassinne E, et al. Antiphospholipid syndrome and other autoimmune diseases associated with chronic intervillositis. Arch Gynecol Obs. (2015) 291:1229-36. doi: 10.1007/s00404-014-3536-6

10. Vardi L, Paterson H, Hung NA. Successful pregnancy following treatment of recurrent chronic histiocytic intervillositis. BMJ Case Rep. (2017) 2017:bcr2016217886. doi: 10.1136/bcr-2016-217886

11. Gordijn SJ, Beune IM, Thilaganathan B, Papageorghiou A, Baschat AA, Baker $\mathrm{PN}$, et al. Consensus definition of fetal growth restriction: a Delphi procedure. Ultrasound Obstet Gynecol. (2016) 48:333-9. doi: 10.1002/uog.15884

12. Gardosi J, Williams A, Hugh O FA. Customised Centile Calculator, GROW Version 2.1.6.1. Gestation Network (2020). Available online at: www.gestation.net (accessed September 29, 2021).

13. Abdulghani S, Moretti F, Gruslin A, Grynspan D. Recurrent massive perivillous fibrin deposition and chronic intervillositis treated with heparin and intravenous immunoglobulin: a case report. J Obstet Gynaecol Can. (2017) 39:676-81. doi: 10.1016/j.jogc.2017.03.089

14. Mattuizzi A, Sauvestre F, André G, Poingt M, Camberlein C, Carles D, et al. Adverse perinatal outcomes of chronic intervillositis of unknown etiology: an observational retrospective study of 122 cases. Sci Rep. (2020) 10:12611. doi: 10.1038/s41598-020-69191-9

15. Nowak C, Joubert M, Jossic F, Masseau A, Hamidou M, Philippe HJ, et al. Perinatal prognosis of pregnancies complicated by placental chronic villitis or contributed toward revision and approved the manuscript for submission.

\section{FUNDING}

This study was funded by Tommy's the Baby Charity.

\section{ACKNOWLEDGMENTS}

The authors would like to acknowledge and thank Dr. Aisha Anwar for her contribution to data collection for this study.

intervillositis of unknown etiology and combined lesions: about a series of 178 cases. Placenta. (2016) 44:104-8. doi: 10.1016/j.placenta.2016.04.017

16. Boog G, Le Vaillant C, Alnoukari F, Jossic F, Barrier J, Muller J-Y. [Combining corticosteroid and aspirin for the prevention of recurrent villitis or intervillositis of unknown etiology]. J Gynecol Obstet Biol Reprod. (2006) 35:396-404. doi: 10.1016/s0368-2315(06)76411-0

17. Reus AD, van Besouw NM, Molenaar NM, Steegers EA, Visser W, de Kuiper RP, et al. An immunological basis for chronic histiocytic intervillositis in recurrent fetal loss. Am J Reprod Immunol. (2013) 70:230-7. doi: 10.1111/aji.12125

18. Bendon RW, Coventry S, Thompson M, Rudzinski ER, Williams EM, Oron AP. Significance of C4d immunostaining in placental chronic intervillositis. Pediatr Dev Pathol. (2015) 18:362-8. doi: 10.2350/14-12-1582-OA.1

19. Scott RE, Greenwood SL, Hayes DJL, Baker BC, Jones RL, Heazell AEP. Effects of hydroxychloroquine on the human placenta-findings from in vitro experimental data and a systematic review. Reprod Toxicol. (2019) 87:50-9. doi: 10.1016/j.reprotox.2019.05.056

20. Williams DJ. Chronic histiocytic intervillositis and treatment to prevent recurrent pregnancy loss. In: Royal College of Obstetricians \& Gynaecologists' (RCOG) Virtual World Congress (2021).

21. Mekinian A, Houfflin-Debarge V, Kolanska K, Cohen J, Abisror N, Bornes $\mathrm{M}$, et al. Antagonists of $\mathrm{TNF} \alpha$ for recurrent miscarriages: 2 Illustrative cases. Eur J Obstet Gynecol Reprod Biol. (2019) 236:263-4. doi: 10.1016/j.ejogrb.2019.02.036

22. Bos M, Harris-Mostert ETMS, van der Meeren LE, Baelde JJ, Williams DJ, Nikkels PGJ, et al. Clinical outcomes in chronic intervillositis of unknown etiology. Placenta. (2020) 91:19-23. doi: 10.1016/j.placenta.2020.01.001

23. Labarrere C, Althabe O. Chronic villitis of unknown aetiology in recurrent intrauterine fetal growth retardation. Placenta. (1987) 8:167-73. doi: 10.1016/0143-4004(87) 90019-1

24. Derricott H, Jones RL, Heazell AEP. Investigating the association of villitis of unknown etiology with stillbirth and fetal growth restriction - a systematic review. Placenta. (2013) 34:856-62. doi: 10.1016/j.placenta.2013.07.003

Conflict of Interest: The authors declare that the research was conducted in the absence of any commercial or financial relationships that could be construed as a potential conflict of interest.

Publisher's Note: All claims expressed in this article are solely those of the authors and do not necessarily represent those of their affiliated organizations, or those of the publisher, the editors and the reviewers. Any product that may be evaluated in this article, or claim that may be made by its manufacturer, is not guaranteed or endorsed by the publisher.

Copyright (c) 2021 Brady, Williams, Batra, Church, Tower, Crocker and Heazell. This is an open-access article distributed under the terms of the Creative Commons Attribution License (CC BY). The use, distribution or reproduction in other forums is permitted, provided the original author(s) and the copyright owner(s) are credited and that the original publication in this journal is cited, in accordance with accepted academic practice. No use, distribution or reproduction is permitted which does not comply with these terms. 